\title{
Brake squeal control with shunted piezoceramics - efficient modelling and experiments
}

\author{
M Neubauer* and R Oleskiewicz \\ Institute of Dynamics and Vibrations, University of Hannover, Hannover, Germany
}

The manuscript was received on 30 March 2007 and was accepted after revision for publication on 10 January 2008.

DOI: 10.1243/09544070JAUTO592

\begin{abstract}
Due to increased interest in comfort features, considerable effort has been spent on the modification of brake prototypes in order to suppress brake squeal. Recently, piezoelectric transducers have been investigated as an innovative way of controlling the vibrations of a brake. This paper presents an efficient mechanical model for the description of a brake with embedded transducers. Different control strategies are discussed and compared. Measurements on a brake test rig validate the obtained results.
\end{abstract}

Keywords: brake squeal, Kirchhoff plate, shunted piezoelectric transducer, negative capacitance, vibration damping, feedback control

\section{INTRODUCTION}

Brake noises with dominating frequencies above $1 \mathrm{kHz}$ are typically termed 'brake squeal'. While these vibrations do not affect the brake function itself, they cause considerable noise. The phenomenon of brake squeal is not wholly understood and brake squeal remains unpredictable. Therefore, brake manufacturers typically reduce the tendency to squeal in a time- and cost-consuming process of designing, building, and testing of prototypes in a mostly empirical way.

Recently, piezoceramic actuators have been studied for an active feedback control of brake vibrations $[\mathbf{1}, 2]$. While this approach shows a very good performance, the requirement of complex sensing devices and amplifiers makes this technique expensive. An alternative approach is piezoelectric shunt damping [3]. This technique has been a very active area of research over the past two decades $[4,5]$. It requires - in the simplest way - only one single actuator, which is shunted with an electric branch. Passive inductance-resistance branches offer high energy dissipation [6], but they require precise tuning to the squealing frequency. Negative capacitance shunts increase the performance of passive LR

*Corresponding author: Institute of Dynamics and Vibrations, University of Hannover, Appelstr. 11, Hannover, D30167, Germany.email: neubauer@ids.uni-hannover.de shunts [7-9]. A synthetic negative capacitance can be realized by a negative impedance converter circuit; hence it requires power for operation.

This work focuses on resonant LR and LRC shunts with a synthetic negative capacitance. Based on a linear, one-dimensional model, the shunted piezoceramic actuator is described by its dynamic, complex stiffness. It can be modelled as a springdamper element in parallel configuration, albeit with frequency-dependent stiffness and damping properties. The second approach is a collocated pair of sensor and actuator with an internal feedback loop. It is described by its complex stiffness in a similar manner to emphasize the likeness between both techniques.

Designing the optimal controller to suppress squealing requires a model of the brake. Because of the high-frequency range of brake vibrations, the models must be kept as simple as possible to be eligible for real-time control. Therefore, multibody systems with a moderate number of degrees of freedom are chosen to simulate the dynamical behaviour of the brake $[\mathbf{1 0}, \mathbf{1 1}]$. They are compromises of precise but time-consuming models, which have to be reduced down to the essential properties. Naturally, these models are not capable of reflecting the dynamics of the brake system in all details. However, they help to gain more insight into the mechanisms that generate brake squeal. 
The transverse vibrations of the brake disc strongly influence squealing; therefore one focus must be placed on modelling the disc. Kirchhoff plate models show a good accordance with measured eigenfrequencies of a disc in the relevant frequency range. Simplest models consider only one mode of the disc with a corresponding frequency that is closest to the squealing frequency [11], but the models can be extended for a multimode description of the disc [12]. The homogeneous plate model is only a rough approximation of the real disc, which typically has a complex geometry. Therefore, the dynamic impedance of the disc model must be adapted in the frequency range by adjusting disc parameters like height and mass per unit density. However, these models do not fit all relevant natural frequencies of the disc with the same precision [12]. Therefore, this paper compares the different boundary conditions for the inner radius of the annular plates that can be found in the literature [13] and determines the conditions that meet all measured natural frequencies best.

This paper compares two techniques to control the squealing, namely a PD (proportional derivative)feedback control and piezoelectric shunt damping with resonant LR and LRC shunts. One focus is placed on the improvement by a negative capacitance and on tuning the shunt parameters. The stability of the brake model is obtained in a complex eigenvalue analysis. Experimental results are conducted that validate the obtained results.

\section{PIEZOELECTRIC MODEL}

The description of the piezoceramic actuator is based on a linear, one-dimensional model, as shown in Fig. 1. Non-linear effects like hysteresis, etc., are not covered by this model. The piezoelectric effect is captured by the fundamental piezoelectric equation

$$
\left[\begin{array}{c}
\sigma \\
D
\end{array}\right]=\left[\begin{array}{cc}
K_{\mathrm{E}} & -e \\
e & \varepsilon_{\mathrm{S}}
\end{array}\right]\left[\begin{array}{l}
S \\
E
\end{array}\right]
$$

This matrix equation couples mechanical properties (stress $\sigma$ and strain $S$ ) with electrical properties (electrical field $E$ and electrical displacement $D$ ). The actuator is described in a mechanical way by its modulus of elasticity $K_{\mathrm{E}}$ and in an electrical way by its permittivity $\varepsilon_{s}$. The piezoelectric constant $e$ couples mechanical and electrical subsystems. Based on this equation the voltage at the electrodes of the piezoceramic can be given as a function of the deformation $x_{\mathrm{p}}$ of the actuator in Laplace domain

$$
U_{\mathrm{p}}(s)=-\frac{1}{1+\left[s C_{\mathrm{p}} Z(s)\right]^{-1}} \frac{c_{33} d_{33}}{C_{\mathrm{p}}} X_{\mathrm{p}}(s)
$$

The voltage depends on the parameters of the actuator (stiffness $c_{33}$, sensitivity $d_{33}$, capacitance $C_{\mathrm{p}}$ ) and on the electrical impedance $Z(s)$, which is connected at the electrodes. Using the piezoelectric transducer as a sensor requires a high electrical impedance of the load, $Z(s) \rightarrow \infty$, so that the charge is kept at the electrodes. From equation (2) it follows that the change in deformation of the transducer is proportional to the voltage

$$
\dot{u}_{\mathrm{p}}(t)=-\frac{c_{33} d_{33}}{C_{\mathrm{p}}} \dot{x}_{\mathrm{p}}(t)
$$

The force that is generated by the piezoceramic actuator consists of two terms

$$
F_{\mathrm{p}}(t)=c_{33} x_{\mathrm{p}}(t)-c_{33} d_{33} u_{\mathrm{p}}(t)
$$

The first part corresponds to the restoring force due

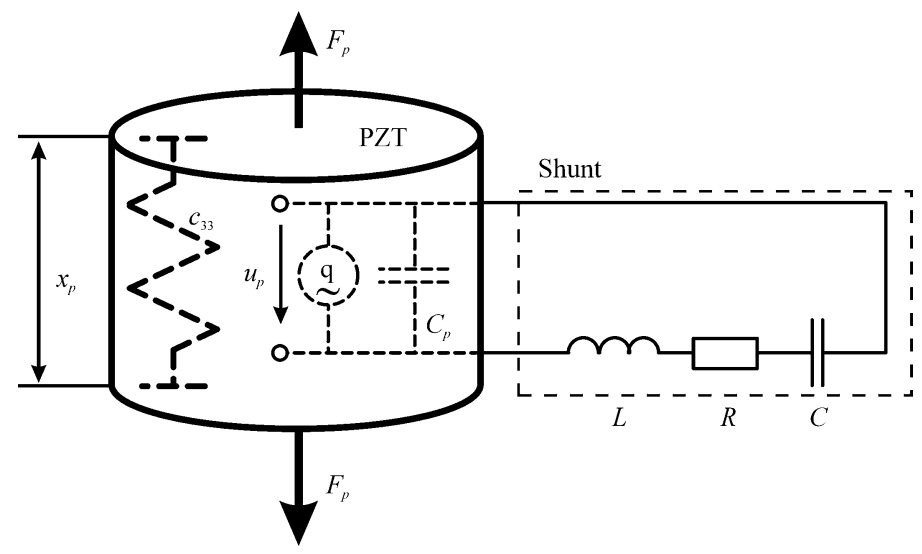

Fig. 1 Model of piezoceramic and the attached LRC shunt 
to the mechanical stiffness of the actuator. The second part is determined by the applied voltage on the electrodes, which is the control voltage of the transducer if utilized as an actuator in a feedback control. If the transducer is shunted by a network with impedance $Z(s)$, the voltage results directly from equation (2). Inserting this expression into equation (4) yields a dependency between the mechanical deformation $x_{\mathrm{p}}$ of the shunted transducer and the resulting piezoforce $F_{\mathrm{p}}$

$F_{\mathrm{p}}(s)=c_{33}\left[1+K^{2} \frac{Z(s)}{Z(s)+\left(s C_{\mathrm{p}}\right)^{-1}}\right] X_{\mathrm{p}}(s), K^{2}=\frac{c_{33} d_{33}^{2}}{C_{\mathrm{p}}}$

where $K$ represents the electromechanical coupling coefficient of the piezoelectric transducer. The voltage at the electrodes is treated as an inner degree of freedom in this representation of the transducer.

\subsection{Shunt damping with LR and LRC branches}

Passive LR shunts consisting of an inductance $L$ and a resistance $R$ are commonly used for piezoelectric shunt damping. The branch impedance reads $Z(s)=$ $R+s L$. This technique requires very precise tuning of the electrical natural frequency which reads

$$
\omega_{\text {elec }}=\frac{1}{\sqrt{L C_{\mathrm{p}}}}
$$

Inserting the impedance of the LR shunt into equation (5) and separating into real and complex parts yields

$$
\begin{aligned}
F_{\mathrm{p}}(\Omega)= & {\left[c_{33}+c_{33} K^{2} \frac{\left(C_{\mathrm{p}} L\right)^{2} \Omega^{4}+\left(4 \zeta^{2}-1\right) C_{\mathrm{p}} L \Omega^{2}}{\left(1-C_{\mathrm{p}} L \Omega^{2}\right)^{2}+4 \zeta^{2} C_{\mathrm{p}} L \Omega^{2}}\right.} \\
& \left.+c_{33} K^{2} \frac{2 \zeta \sqrt{C_{\mathrm{p}} L} \Omega}{\left(1-C_{\mathrm{p}} L \Omega^{2}\right)^{2}+4 \zeta^{2} C_{\mathrm{p}} L \Omega^{2}} j\right] X_{\mathrm{p}}(\Omega)
\end{aligned}
$$

The damping ratio of the electrical LR branch is denoted as $\zeta$. The force dependency in equation (7) can be considered as the dynamical, complex stiffness of the shunted transducer which is valid for the assumption of a harmonic excitation with frequency $\Omega$. Mechanically it can be represented by a spring-damper element with frequency-dependent stiffness and damping properties. The corresponding parameters $c_{\text {res }}, d_{\text {res }}$ are determined by equalizing real and imaginary parts for the shunted transducer and the spring-damper element

$$
\begin{aligned}
& c_{\text {res }}=c_{33}+c_{33} K^{2} \frac{\left(C_{\mathrm{p}} L\right)^{2} \Omega^{4}+\left(4 \zeta^{2}-1\right) C_{\mathrm{p}} L \Omega^{2}}{\left(1-C_{\mathrm{p}} L \Omega^{2}\right)^{2}+4 \zeta^{2} C_{\mathrm{p}} L \Omega^{2}} \\
& d_{\text {res }}=c_{33} K^{2} \frac{2 \zeta \sqrt{C_{\mathrm{p}} L}}{\left(1-C_{\mathrm{p}} L \Omega^{2}\right)^{2}+4 \zeta^{2} C_{\mathrm{p}} L \Omega^{2}}
\end{aligned}
$$

The shunt inductance $L$ and the damping ratio $\zeta$ influence both the real part and the imaginary part of the complex stiffness. Therefore, the dynamic stiffness and damping cannot be adjusted separately.

Previous publications mention the increase in damping performance of a shunted piezoelectric transducer with a negative capacitance. In the following, the external capacitance $C$ in the LRC branch is normalized to the piezocapacitance $C_{\mathrm{p}}$

$$
\delta=\frac{C_{\mathrm{p}}}{C}
$$

A negative capacitance shunt is indicated by a negative value of $\delta$. The natural frequency of the piezoelectric system is influenced by the negative capacitance. A positive capacitance increases the frequency, while a negative value leads to a reduction

$$
\omega_{\text {elec }}=\sqrt{\frac{1+\delta}{L C_{\mathrm{p}}}}
$$

The dynamic stiffness and damping of a transducer with negative capacitance shunt can be obtained in the same way as for the LR shunt. It results in lengthy equations for the complex stiffness, which are not given here. However, it is shown in reference [14] that the dissipated energy of LRC shunts outperforms the passive LR shunts

$$
E_{\mathrm{diss}, \mathrm{LRC}}=\frac{1}{1+\delta} E_{\mathrm{diss}, \mathrm{LR}}
$$

Performance is therefore maximized by tuning the capacitance ratio $\delta \rightarrow-1$, which is the stability boundary of the electrical branch. In practical applications, the capacitance value must keep a safety margin to the stability boundary, e.g. $\delta=-0.9$.

\subsection{PD-feedback control}

A feedback strategy to stabilize a disc brake is suggested in reference [1]. This technique requires at least one additional sensor. In this context, a simple 
PD-feedback with a collocated pair of identical actuator and sensor is discussed. The voltage on the sensor-transducer is fed back with constant gains for proportional $\left(g_{\mathrm{p}}\right)$ and derivative feedback $\left(g_{\mathrm{d}}\right)$ and applied at the electrodes of the actuator

$$
u_{\text {actuator }}=g_{\mathrm{p}} u_{\text {sensor }}+g_{\mathrm{d}} \dot{u}_{\text {sensor }}
$$

The collocated pair of sensor and actuator transducer with a local feedback loop can be expressed as a complex stiffness. Inserting the control voltage according to equation (12) and the sensor voltage according to equation (3) into equation (4) results in the following force of the pair of actuator-sensor with a local feedback loop

$$
\begin{aligned}
F_{\mathrm{p}} & =\left(1+K^{2}\right) c_{33} x_{\mathrm{p}}+c_{33} x_{\mathrm{p}}+c_{33} d_{33} u_{\text {actuator }} \\
& =c_{33}\left[2+K^{2}\left(1+g_{\mathrm{p}}\right)\right] x_{\mathrm{p}}+c_{33} K^{2} g_{\mathrm{d}} \dot{x}_{\mathrm{p}}
\end{aligned}
$$

This expression can easily be modified for a sensoractuator pair with different transducers. Contrary to the piezoelectric shunt damping technique, the stiffness does not depend on the frequency. Real and imaginary parts can be tuned separately by feedback gains $g_{\mathrm{p}}$, $g_{\mathrm{d}}$.

\section{BRAKE MODEL}

The dynamical behaviour of the brake is described as a multibody system with a flexible disc model. Such multibody systems are a rough approximation of real brake systems, which cannot model every phenomenon of brake squeal in detail. However, multibody systems give better insight into the basic mechanisms that lead to brake squeal and they allow an efficient calculation, which is required for real-time control.

The brake model is depicted in Fig. 2. It consists of the brake disc, two brake pads, and the embedded transducer plus attached shunt or feedback loop. System parameters like mass, stiffness, and damping are adjusted so that measured frequencies of the brake are met by the model. The PD-feedback loop with collocated sensor-actuator pair is modelled by the complex stiffness according to section 2.1. This is also possible for shunted transducers, but in this case the resulting damping and stiffness matrices of the overall system are frequency-dependent. A more convenient way of modelling shunted transducers is to treat the voltage at the electrodes as an additional degree of freedom. This increases the total degrees of freedom (DOF) of the system by one, but with the outcome of constant system matrices. An LRCshunted transducer placed between DOFs $x_{\mathrm{i}}$ and $x_{\mathrm{j}}$ of a multibody is incorporated into the system matrices of the overall system by adding the following submatrices according to reference [3]

$$
\begin{aligned}
& {\left[\begin{array}{ccc}
0 & 0 & 0 \\
0 & 0 & 0 \\
-c_{33} d_{33} L & c_{33} d_{33} L & C_{\mathrm{p}} L
\end{array}\right]\left[\begin{array}{c}
\ddot{x}_{\mathrm{i}} \\
\ddot{x}_{\mathrm{j}} \\
\ddot{u}_{\mathrm{p}}
\end{array}\right]} \\
& +\left[\begin{array}{ccc}
0 & 0 & 0 \\
0 & 0 & 0 \\
-c_{33} d_{33} R & c_{33} d_{33} R & C_{\mathrm{p}} R
\end{array}\right]\left[\begin{array}{c}
\dot{x}_{\mathrm{i}} \\
\dot{x}_{\mathrm{j}} \\
\dot{u}_{\mathrm{p}}
\end{array}\right] \\
& +\left[\begin{array}{ccc}
c_{33} & -c_{33} & c_{33} d_{33} \\
-c_{33} & c_{33} & -c_{33} d_{33} \\
\frac{c_{33} d_{33}}{C} & -\frac{c_{33} d_{33}}{C} & 1+\delta
\end{array}\right]\left[\begin{array}{l}
x_{\mathrm{i}} \\
x_{\mathrm{j}} \\
u_{\mathrm{p}}
\end{array}\right]=\left[\begin{array}{l}
0 \\
0 \\
0
\end{array}\right]
\end{aligned}
$$
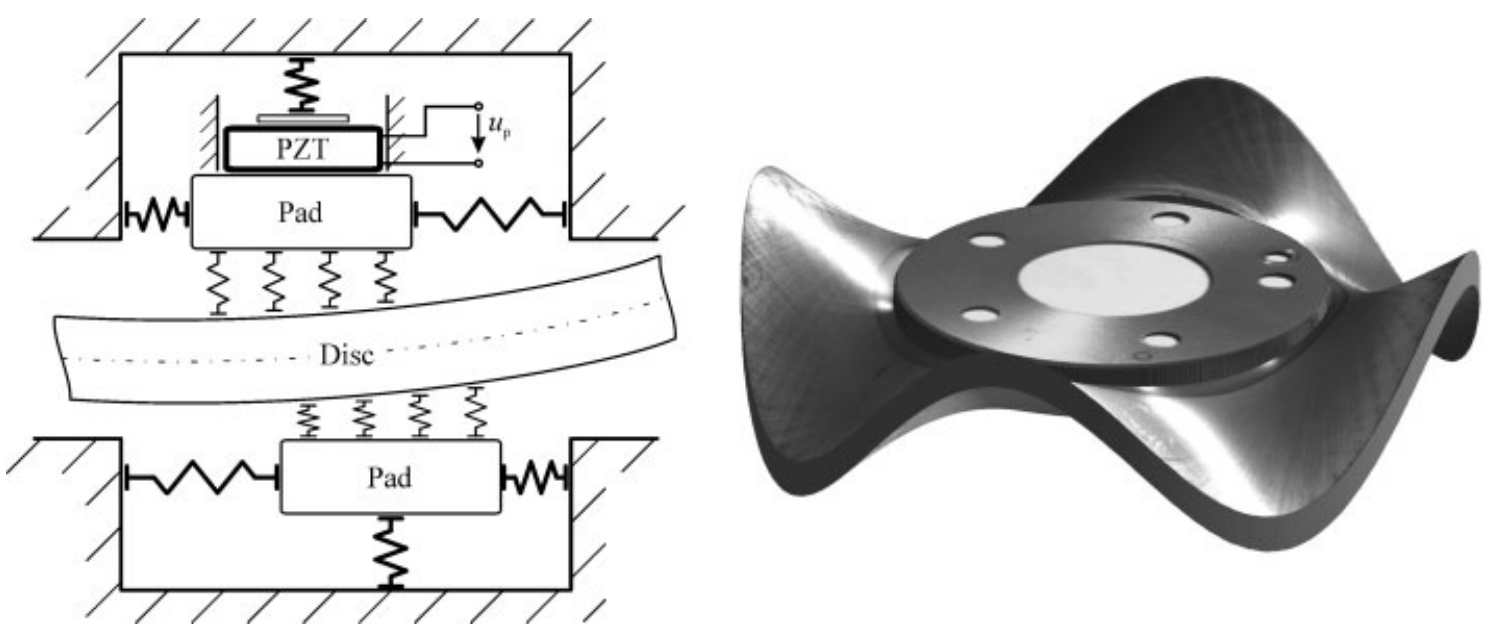

Fig. 2 Multibody system of the brake and $4 / 1$ eigenmode of the disc 
In this case the vector of generalized variables consists of seven mechanical DOFs and the voltage as an electrical DOF

$$
x=\left[\begin{array}{c}
x_{\text {mech }} \\
u_{\mathrm{p}}
\end{array}\right]
$$

\subsection{Disc model}

The brake disc is described as a flexible annular Kirchhoff plate with an inner radius $r_{\mathrm{i}}$ and outer radius $r_{\mathrm{a}}$. Brake squeal usually occurs at low rotating velocities; therefore rotational inertia and shear forces can be neglected and the equation for transverse oscillations $w(r, \varphi, t)$ reads

$$
\begin{aligned}
& \rho h\left[\frac{\partial^{2}}{\partial t^{2}} w(r, \varphi, t)+2 \Omega \frac{\partial^{2}}{\partial \varphi \partial t} w(r, \varphi, t)\right] \\
& +D_{\mathrm{disc}} \nabla^{4} w(r, \varphi, t)=q(r, \varphi, t)
\end{aligned}
$$

with mass per unit area $\rho h$, external transverse pressure load $q(r, \varphi, t)$ as a function of position (radius $r$, angle $\varphi$ ) and time $t$, the bending stiffness $D_{\text {disc, }}$ and the differential operator $\nabla$. The mode shapes and eigenfrequencies are obtained by solving the eigenvalue problem analytically. The vibration of the disc can be discretized and separated in time and space

$$
\begin{aligned}
& w(r, \varphi, t) \\
& =\sum_{m, n=1}^{\infty} R_{m, n}(r)\left[A_{m, n}(t) \cos (m \varphi)+B_{m, n}(t) \sin (m \varphi)\right]
\end{aligned}
$$

Parameters $m$ and $n$ represent the number of nodal diameters and circles. The radial shape functions $R_{m, n}$, which are sums of Bessel functions, result from the eigenvalue problem, the circumferential part are sine and cosine functions, and $A_{m, n}(t)$ and $B_{m, n}(t)$ are time functions.

The height of the disc is typically tuned in order to adapt the eigenfrequency of the disc model to a measured frequency response. This tuning is necessary because a real vented brake disc differs from the homogeneous Kirchhoff plate. One eigenfrequency of the disc can always be tuned exactly to one measured frequency by choosing an appropriate height. However, changing the height shifts all eigenfrequencies of the disc by the same percentage; i.e. the ratio of different disc eigenfrequencies cannot be influenced by the height. Therefore, the height is chosen by a least error fit of all eigenfrequencies in the relevant frequency range. It cannot be expected that all eigenfrequencies are perfectly matched.

The choice of boundary conditions affects the radial shape functions $R_{m, n}$ as well as the eigenfrequencies of the disc. Particularly, the ratio of different disc eigenfrequencies is influenced by the boundary conditions of the disc. Comparing measured and calculated eigenfrequencies of the disc, the boundary condition that fits the frequency ratio of all relevant modes best can be found. Obviously, the choice of boundary conditions also affects the optimal height of the disc model. Apparently, the boundary condition for the outer radius of the disc is free but the choice for the inner radius of the disc is not straightforward. Different conditions are suggested in the literature: clamped, simply supported, and free.

Figure 3 shows the measured frequency response of a vented brake disc in the frequency range up to $8 \mathrm{kHz}$. The disc is excited by a shaker and the oscillations are measured by a scanning laser vibrometer. A Kirchhoff disc with identical inner and outer radii is modelled and the eigenfrequencies are calculated for the above-mentioned boundary conditions for the inner ring. During calculations, the disc height is kept as a variable. Subsequently, the mean error of all eigenfrequencies in the range between 1 and $8 \mathrm{kHz}$ is determined versus the disc height according to the following equation

$$
\Delta f=\sum_{i=1}^{n}\left(\left|f_{\mathrm{i}, \text { meas }}-f_{\mathrm{i} \text {,calc }}\right| / f_{\mathrm{i} \text {,meas }}\right) / n
$$

where $f_{\mathrm{i} \text {,meas }}$ and $f_{\mathrm{i}, \text { calc }}$ are the measured and calculated eigenfrequencies of the disc. The result

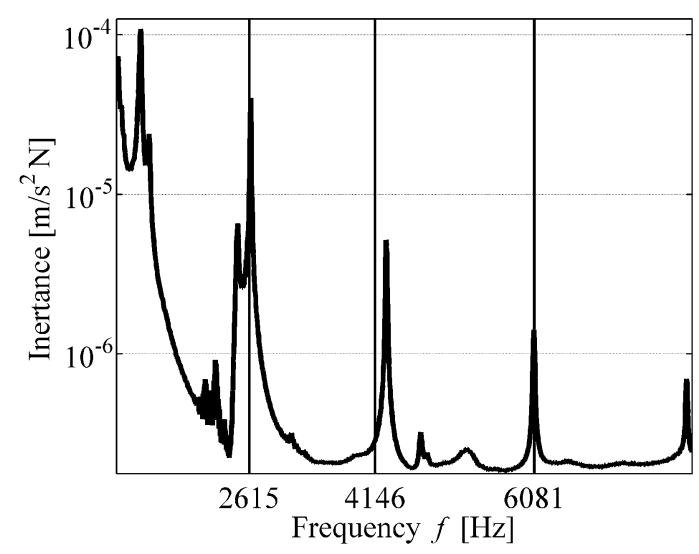

Fig. 3 Measured frequency response of the brake disc and calculated eigenfrequencies 


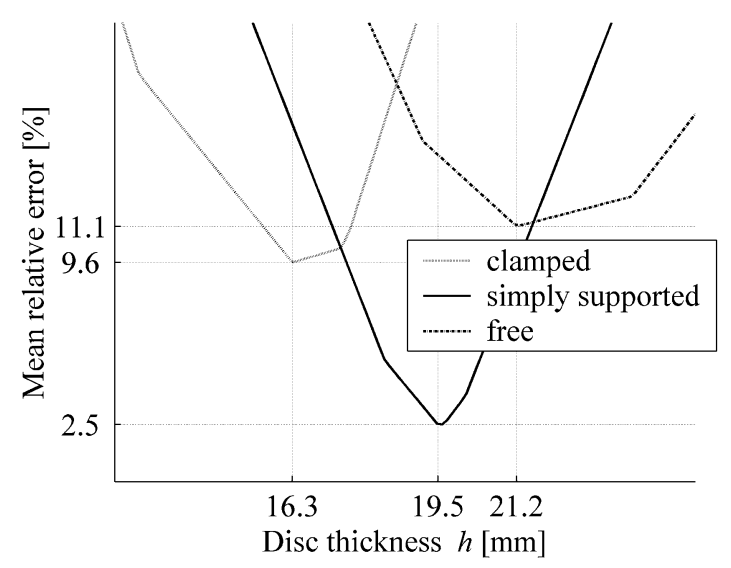

Fig. 4 Mean error of calculated eigenfrequencies versus disc height for different boundary conditions

is presented in Fig. 4. The function of the mean error is stepwise linear. For each boundary condition for the inner radius (clamped, simply supported, and free), one optimal height $h_{\text {opt }}$ can be found that minimizes the relative error in frequencies. The optimal height depends on the boundary condition. It is plausible that the clamped boundary condition, which introduces the most stiffness, results in the smallest height.

None of the assumed boundary conditions results in a perfect fit of all eigenfrequencies in the considered frequency range. However, relative error is minimized with the boundary condition 'simply supported', with a mean frequency error of less than 2.5 per cent compared with the measured frequencies. The calculated eigenfrequencies of the disc with this boundary condition is marked in Fig. 3. A second best fit of eigenfrequencies is obtained with the boundary condition 'clamped inner ring' with a mean error of 9.6 per cent, followed by 'free inner ring' with an 11.1 per cent difference. It must be mentioned that these results are not general; using a disc with vastly different geometry may lead to different results. However, these measurements are performed on a standard automotive brake disc, and the result should be valid for the majority of similar disc types.

As a result of the above results, the disc is modelled with boundary conditions 'simply supported' at the inner ring and 'free' at the outer ring. However, in the following calculations only the disc mode $m=4, n=1$ is considered for disc vibrations, which is closest to the measured squealing frequency. Therefore, vibrations of the disc are described by two coordinates, $A(t)$ and $B(t)$. This is a plausible simplification, as the shunted transducer must be tuned to one single mechanical mode, and the remaining modes are not damped by the transducer because of the small frequency bandwidth with high energy dissipation.

The contact between the disc and brake pads is simplified as a sector-shaped contact area and is described by Heaviside functions $H(r), H(\varphi)$. Oscillations of the pads in the in-plane direction with $x_{\text {pad,i }}$ shift the contact area

$$
\begin{aligned}
& H(r)= \begin{cases}1, & r_{1}<r<r_{2} \\
0, & \text { else }\end{cases} \\
& H(\varphi)= \begin{cases}1, & -\beta+x_{\mathrm{pad}, \mathrm{i}}<\varphi<\beta+x_{\mathrm{pad}, \mathrm{i}} \\
0, & \text { else }\end{cases}
\end{aligned}
$$

The contact is modelled as a layer with constant stiffness and damping properties. Oscillations of the disc and pads create a pressure distribution between the disc and pads, which results in a friction force. A constant coefficient of friction $\mu$ is assumed.

\section{STABILITY ANALYSIS}

The stability of the linear electromechanical multibody system with an embedded transducer is obtained by solving the matrix eigenvalue problem. The maximum real part $\lambda_{\max }$ of all eigenvalues determines the stability. If the real parts of all eigenvalues are negative, $\lambda_{\max }<0$, the system is mainly asymptotically stable. A positive maximum real part indicates instability and the onset of vibrations, which may lead to squealing. The phenomenon of brake squeal is thoroughly discussed in the literature [15]; it is mainly explained as selfexcited vibrations, which are caused by a decreasing friction characteristic over relative speed and by mode coupling because of a moving load. With the obtained complex eigenvalues, the noise index $\alpha$ [16] of the brake model is defined as

$$
\alpha=\frac{\Re}{\sqrt{\Re^{2}+\Im^{2}}}
$$

This index specifies the growth rate of brake vibrations. It is only valid for small vibration amplitudes. In reality, the vibration amplitudes depend on nonlinear effects like stick-slip limit cycles.

Figure 5 depicts the noise index of the brake without shunting of the transducer. In the linear model, the non-conservative forces are proportional 


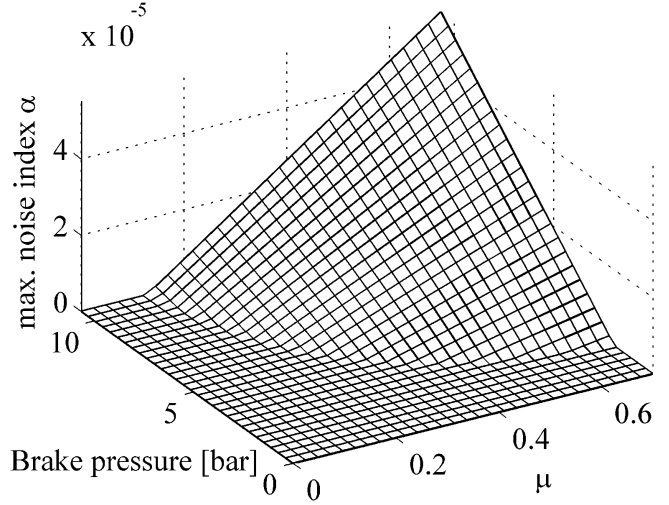

Fig. 5 Noise level of the brake model versus brake pressure and coefficient of friction

to the product of brake pressure and coefficient of friction (COF) $\mu$. Because of damping in the system, the brake model is stable for low pressures and COF, which is indicated by a negative noise index. However, for realistic values for pressure and COF the noise index is positive.

\subsection{PD-feedback}

Feedback gains $g_{\mathrm{p}}, g_{\mathrm{d}}$ must be optimized with the aim of stabilizing the brake. This is equivalent to minimizing the maximum real part $\lambda_{\max }$ of the controlled system. For this purpose, the feedback gains $g_{\mathrm{p}}, g_{\mathrm{d}}$ are varied in a broad range, and the resulting complex stiffness of the shunted transducer according to equation (12) is calculated. Complex eigenvalues of the system are calculated and the maximum real part is shown in Fig. 6 versus feedback gains.

The area with a negative maximum real part corresponds to a stable brake. For optimal feedback, the maximum real part can be reduced to

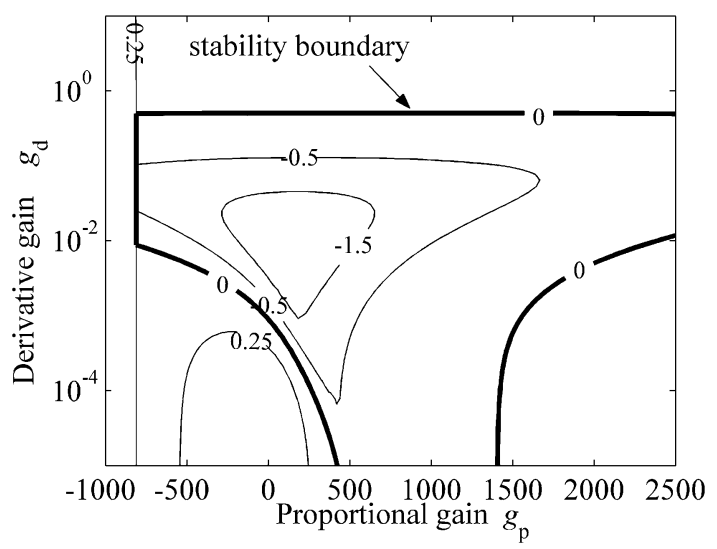

Fig. 6 Stability graph of feedback control versus feedback gains $\lambda_{\max }=-1.5$. The required feedback gains can be seen in Fig. 6. A good result can already be obtained by a feedback of the derivative part only, which adds damping to the system.

\subsection{LRC and LR shunt damping}

Stability analysis for the shunted piezoelectric transducer is performed in a similar way. The shunt parameters $L, R, \delta$ are varied simultaneously and the corresponding complex eigenvalues are calculated. The maximum real part is given in Fig. 7 versus normalized shunt parameters. External inductance is normalized to the optimal value for a tuned shunt.

The frequency dependency of these resonant shunts can be seen clearly; the inductance $L$, which influences the electrical natural frequency, must be tuned very precisely. Up to some point, additional damping in the network increases the frequency bandwidth with a stable system, but choosing the resistance too high will cancel the stabilizing effect altogether. The performance increase due to a negative capacitance is clearly visible (notice that the slice with $\delta=0$ is a passive LR shunt). While the damping ratio for passive LR shunts must be kept very small, semi-active LRC shunts offer the broadest stabilized frequency range for slightly larger damping ratios.

\section{MEASUREMENTS}

For a validation of the obtained results, an automotive disc brake has been modified. The brake prototype is depicted in Fig. 8. Three identical stack actuators from the company MARCO with capacitance $C_{\mathrm{p}}=23 \mathrm{nF}$ are placed between the piston and

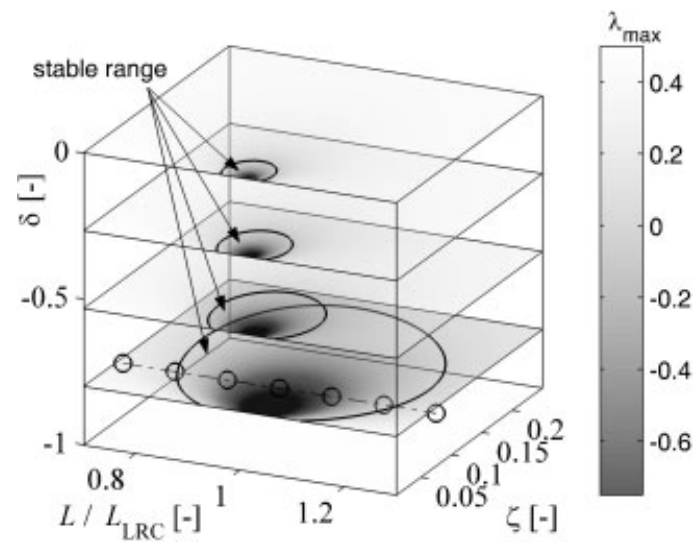

Fig. 7 Stability graph of the the LRC branch versus normalized shunt parameters 


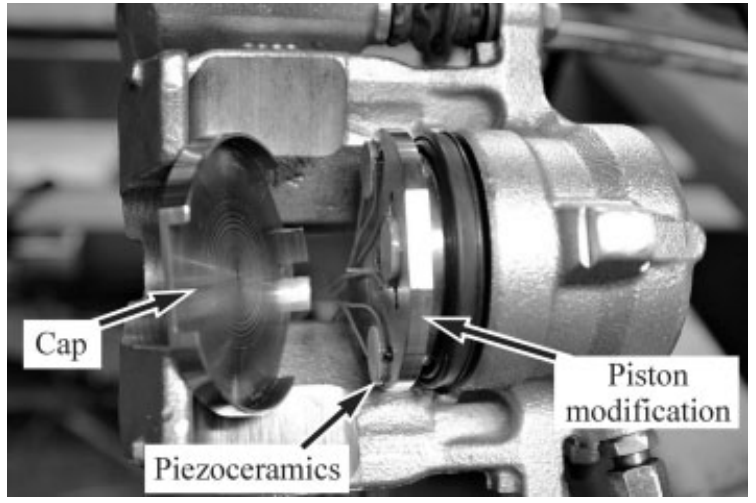

Fig. 8 Photo of the modified brake

the inboard brake pad and connected in parallel for shunt damping. The normal pressure on the piezoelectric transducer consists of the brake pressure and a superimposed sinusoidal-like fluctuating part with the frequency of squealing. For shunt damping, all three transducers are connected in parallel to one single LRC branch; for the feedback control, one transducer can be used as a sensor while the others are actuators. The piezoelectric transducers are protected against shear forces by a cap construction.

The brake prototype is examined on a brake test rig at the Institute of Dynamics and Vibrations. The test rig allows automatic brake sequences with a control of the disc speed, brake torque, and disc temperature. Sound radiation is measured by a microphone during measurements. Squealing occurs mostly at low disc speeds with $20-25 \mathrm{r} / \mathrm{min}$ and brake pressures in the range of 10 bar.

Controlling the brake by an active feedback has been shown in reference [2], where optimal feedback gains are established. Immediately after turning the control on, the vibrations of the disc are suppressed and the brake remains silent, until the control is turned off. A maximum control voltage of $50 \mathrm{~V}$ is required for stabilizing the brake.

For realizing an LRC shunt a negative capacitance board has been built with one single high-voltage OPA445 operational amplifier as an impedance converter (see Fig. 9). Negative capacitance values can be gained by changing the amplification factor of the amplifier. A tuneable shunt resistance was incorporated into the circuit; the inductance is connected externally. A voltage supply of $\pm 40 \mathrm{~V}$ is applied to the board. During measurements at the brake, the power consumption was not significantly greater than during the idle state.

The frequency dependency of LR and LRC shunts are analysed by the following procedure. Brake pressure and disc speed are adjusted until a constant and tonal squealing occurs. The optimum shunt inductance is calculated from the measured squealing frequency according to equation (10). Afterwards,

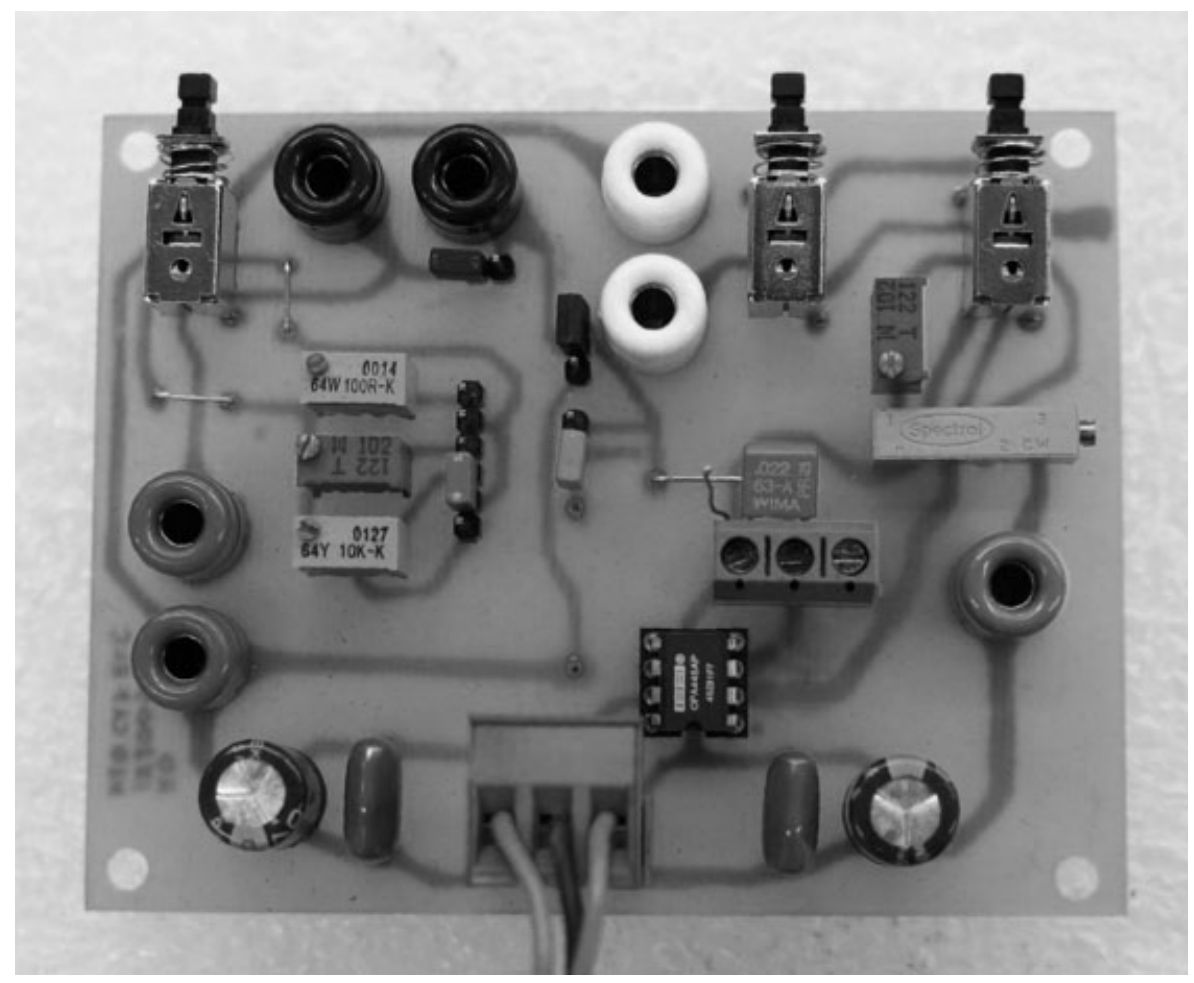

Fig. 9 Negative capacitance board 
the shunt is connected and disconnected periodically in 10-second intervals. After each connection the shunt inductance is increased stepwise. At the beginning of a measurement the inductance value is set to a value smaller than the optimal one and at the end of one measurement the inductance value is higher than the optimal one. In the middle of the measurements the inductance is set optimally. One whole measurement lasts up to 3 minutes. They are repeated for different LR and LRC shunts.

Sound pressure is recorded with a microphone and the mean sound pressure level (SPL) in $\mathrm{dB}$ for each 10 seconds of connection and disconnection is calculated. Figure 10 depicts the time history of the recorded sound pressure for one connection of an LRC shunt. During the 10 seconds of connection the sound pressure is immediately reduced to a constant, low state. After disconnection of the LRC shunt the squealing reappears. The reduction in SPL between the connected shunt and disconnected shunt is shown in Fig. 11. Each interval of connection and disconnection results in one measurement point; they are plotted versus the inductance ratio for LR and LRC shunts. The measurements show a good accordance with the analytical results. Calculated stability boundaries for optimal tuned shunt (inductance and resistance) are included in the figure. The boundary for a negative capacitance shunt with $\delta=-0.86$ shows a perfect agreement with the predicted result. The measurements with $\delta=-0.66$ and $\delta=0$ (passive LR shunt) have a smaller stabilized frequency range than predicted. This is due to the fact that the internal losses in the electrical elements are higher than the required optimal damping ratio, so that the optimal damping ratio could not be set during measurements.

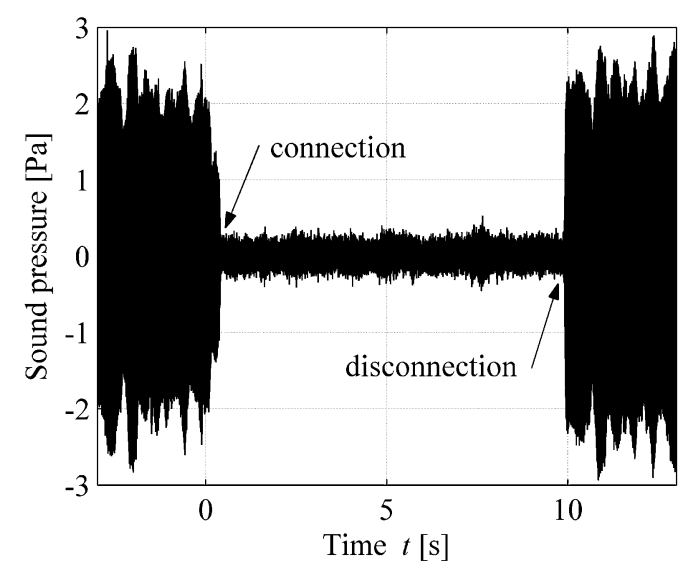

Fig. 10 Time history of sound pressure with the LRC branch

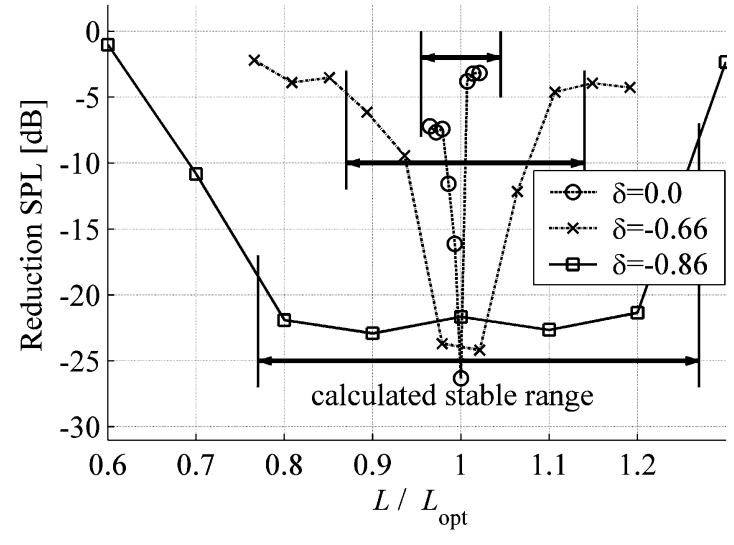

Fig. 11 Reduction of SPL versus normalized inductance and capacitance ratio

All types of shunts are capable of suppressing squealing when optimally tuned. The negative capacitance shunts outperform the passive LR shunts because of the strongly increased frequency bandwidth in which the brake can be stabilized. Measurements prove the binary character of squealing: either the squealing is totally suppressed, which leads to a SPD reduction of approximately $20-25 \mathrm{~dB}$, or the squealing is not influenced, with a reduction of less than $5 \mathrm{~dB}$.

As a concluding remark it must be noted that not all squeal occurrences could be suppressed during measurements. Possible reasons are in-plane oscillations of the disc, which are not detected and influenced by the transducers in the current design. Further, the orientation of the disc mode cannot be influenced by the actuators. Energy out-take is only possible if the vibration nodes are not in the contact area of the disc and brake pads.

\section{CONCLUSIONS}

This paper presents two innovative techniques involving piezoelectric transducers to control brake squeal: a local feedback control with collocated piezoelectric transducers and piezoelectric shunt damping. An efficient modelling of disc brakes and shunted transducers is proposed, which is utilized to determine an optimal feedback loop and optimal shunt parameters.

Stability analysis provides the robustness of passive LR and semi-active LRC shunts for variations of the squealing frequency. A negative capacitance shunt significantly increases the frequency range with a stabilized brake, which is necessary for a robust performance. A validation of the obtained 
results is performed by measurements on a modified disc brake.

\section{REFERENCES}

1 von Wagner, U., Hochlenert, D., Jearsiripongkul, T., and Hagedorn, P. Active control of brake squeal via 'smart pads'. SAE technical paper 2004-01-2773, 2004.

2 Neubauer, M., Neuber, C.-C., and Popp, K. Control of stick-slip vibrations. In IUTAM Symposium on Vibration Control of Nonlinear Mechanisms and Structures, Munich, 2005, pp. 223-232.

3 Neubauer, M., Kröger, M., and Oleskiewicz, R. Suppression of brake squeal using piezoceramics. In Proceedings of the International Conference on Vehicle Braking Technology, Braking 2006, York, 79 May 2006, pp. 254-263 (ISBN 085316 245X).

4 Lesieutre, G. A. Vibration damping and control using shunted piezoelectric materials. Shock Vibr. Dig., 1998, 30, 187-195.

5 Moheimani, S. O. R. A survey of recent innovations in vibration damping and control using shunted piezoelectric transducers. IEEE Trans. on Control Systems Technol., 2003, 11(4), 482-494.

6 Hagood, N. W. and von Flotow, A. H. Damping of structural vibrations with piezoelectric materials and passive electrical networks. J. Sound Vibr., 1991, 146(2), 243-268.

7 Tang, J. and Wang, K. W. Active-passive hybrid piezoelectric networks for vibration control: comparisons and improvement. Smart Mater. Structs, 2001, 10, 794-806.

8 Neubauer, M., Oleskiewicz, R., Popp, K., and Krzyzynski, T. Optimization of damping and absorbing performance of shunted piezoelements utilizing negative capacitance. J. Sound Vibr., 2006, 298, 84-107.

9 Behrens, S., Fleming, A. J., and Moheimani, S. 0. R. A broadband controller for shunt piezoelectric damping of structural vibration. Smart Mater. Structs, February 2003, 12(1), 36-48.

10 Rudolph, M. and Popp, K. Brake squeal. In Detection, utilization and avoidance of nonlinear dynamical effects in engineering applications (Ed. K. Popp), Shaker, Aachen, 2001, pp. 197-225.

11 Hagedorn, P. Modeling disk brakes with respect to squeal. In Proceedings of the 17th International Congress of Mechanical Engineering (COBEM 2003), São Paulo, Brazil, 10-14 November 2003.

12 Jearsiripongkul, T. and Hagedorn, P. Parameter estimation of the disk in a floating caliper disc brake model with respect to squeal. In the GAMM Annual Meeting, 2004, vol. 4, issue 1, pp. 93-94.

13 Boennen, D. and Walsh, S. Investigations of analytical beam and annular plate models for automotive disc brake vibration. In Proceedings of the International Conference on Vehicle Braking

Technology, Braking 2006, York, 7-9 May 2006, pp. 264-274 (ISBN 085316 245X).

14 Oleskiewicz, R., Neubauer, M., and Krzyzynski, T. Vibration control with shunted piezoceramics - the energy balance. In Mechatronics 2006, Heidelberg, 2006.

15 Kinkaid, N. M., O'Reilly, O., and Papadopoulos, P. Automotive disc brake squeal. J. Sound Vibr., 2003, 267, 105-166.

16 Yuan, Y. A study of the effects of negative frictionspeed slope on brake squeal. In Proceedings of the ASME Design Engineering Conference, Boston, vol. 3, 1995, part A, pp. 1135-1162.

\section{APPENDIX}

\section{Notation}

$c_{\text {res }}$

$c_{33}$

C

$C_{\text {ps }}$

$d_{\text {res }}$

$d_{33}$

$D$

$D_{\text {disc }}$

e

E

$E_{\text {diss,LRC }}$

$F_{\mathrm{p}}$

$g_{d}$

gp

K

$K_{\mathrm{E}}$

$L$

$r_{\mathrm{a}}$

$r_{\mathrm{i}}$

$R$

$R_{m, n}$ $S$

$u_{\text {actuator }}$

$u_{\mathrm{p}}$

$u_{\text {sensor }}$

$w$ resulting stiffness of the shunted piezoelectric transducer mechanical stiffness of the piezoelectric transducer capacitance value capacitance of the piezoelectric transducer resulting damping value of the shunted piezoelectric transducer sensitivity of the piezoelectric transducer electrical displacement bending stiffness of the brake disc piezoelectric constant electrical field dissipated energy per vibration period of the LRC-shunted piezoelectric transducer force generated by the piezoelectric transducer derivative feedback gain proportional feedback gain electromechanical coupling coefficient of the piezoelectric transducer modulus of elasticity inductance value outer radius of the brake disc inner radius of the brake disc resistance value radial mode shape strain voltage at the actuator voltage at the electrodes of the piezoelectric transducer voltage at the sensor transverse deformation of the brake disc 


$\begin{array}{llll}x_{\mathrm{p}} & \text { mechanical deformation of the } & \varepsilon_{\mathrm{S}} & \text { permittivity } \\ & \text { piezoelectric transducer } & \zeta & \text { damping ratio of the electrical network } \\ Z(s) & \text { impedance of the electrical } & \mu & \text { coefficient of friction } \\ & \text { network } & \sigma h & \text { mass per unit area } \\ & \text { noise index } & \sigma & \text { stress } \\ \alpha & \text { angle of the contact area } & \omega_{\text {elec }} & \text { electrical natural frequency } \\ \beta & \text { capacitance ratio } & \Omega & \text { excitation frequency }\end{array}$

